\title{
Phosphorylation of p53 at threonine 155 is required for Jab1-mediated nuclear export of p53
}

\author{
Eun-Woo Lee ${ }^{1}$, Wonkyung $\mathrm{Oh}^{2}$, Hosung Paul Song ${ }^{3} \mathcal{E}$ Won Kon Kim ${ }^{1, *}$ \\ ${ }^{1}$ Metabolic Regulation Research Center, Korea Research Institute of Bioscience and Biotechnology (KRIBB), Daejeon 34141 , ${ }^{2}$ DNA Repair \\ Research Center, Chosun University School of Medicine, Gwangju 61452, ${ }^{3}$ Korea International School, Seongnam 13543, Korea
}

The Jun activation-domain binding protein 1 (Jab1) induces p53 nuclear export and cytoplasmic degradation, but the underlying mechanism is poorly understood. Here, we show that phosphorylation at the threonine 155 residue is essential for Jab1-mediated p53 nuclear export. Jab1 stimulated phosphorylation of p53 at T155 was inhibited by curcumin, an inhibitor of COP9 signalosome (CSN)-associated kinases. The T155E mutant, which mimics phosphorylated p53, exhibited spontaneous cytoplasmic localization in the absence of Jab1. This process was prevented by leptinomycin B (LMB), but not by curcumin. The substitution of threonine 155 for valine (T155V) abrogated Jab1-mediated p53 nuclear export, indicating that phosphorylation at this site is essential for Jab1-mediated regulation of $\mathrm{p53}$. Although $\mathrm{T} 155 \mathrm{E}$ can be localized in the cytoplasm in the absence of Mdm2, the translocation of T155E was significantly enhanced by ectopic $\mathrm{Hdm} 2$ expression. Our data suggests that Jab1-mediated phosphorylation of p53 at Thr155 residue mediates nuclear export of p53. [BMB Reports 2017; 50(7): 373-378]

\section{INTRODUCTION}

p53 is a master transcriptional factor called a genome gatekeeper, which suppresses cell growth and tumor formation, and induces cellular senescence (1-3). Since exposure to various oncogenic stresses activates p53, leading to cell cycle arrest or apoptosis, the turn-on process of p53 is a key step in maintaining genomic integrity, and preventing cells from transforming into cancer cells (4-7). Since the transcriptional role of p53 is to keep cells from growing unchecked under DNA damaging conditions, the toxicity of p53 should be suppressed under normal conditions. This is accomplished by the human double minute 2 ( $\mathrm{Hdm} 2$, or $\mathrm{Mdm} 2$ in mice) E3 ligase,

*Corresponding author. Tel: +82-42-860-4265; Fax: +82-42-8604149; E-mail: wkkim@kribb.re.kr

https://doi.org/10.5483/BMBRep.2017.50.7.077

Received 11 May 2017, Revised 24 May 2017, Accepted 25 May 2017

Keywords: COP9 signalosome, Curcumin, Hdm2, Jab1, p53 which continually induces the degradation of p53 $(5,6,8)$.

The Jun activation-domain binding protein 1 (Jab1) is also called CSN5, being a fifth member of COP9 signalosome (CSN) complexes (9). One important function of Jab1 is its ability to mediate nuclear export and/or degradation of its interacting proteins. For example, Jab1 couples these two processes for the Smad7, ER, cyclin E and West-Nile virus capsid protein and p53 (9-19). The detailed biochemical mechanism of how Jab1 mediates nuclear export and/or degradation has not been fully elucidated. However, Jab1 knockout mice displayed increased levels of p53, p27 and cyclin $E$, supporting the physiological importance of Jab1 involvement in regulating the aforementioned proteins (20).

In this study, we identify how Jab1 mediates p53 cytoplasmic translocation. Jab1 induces phosphorylation at Thr155 of p53 via CSN-associated kinases, and stimulates p53 cytoplasmic localization. Phosphorylated p53 can be exported to the cytoplasm through a CRM1-dependent nuclear export system. This process is independent of $\mathrm{Hdm} 2$, but can be facilitated by $\mathrm{Hdm} 2$ overexpression.

\section{RESULTS}

Curcumin prevents Jab1-mediated p53 nuclear export Previously, we noted that p53 could be translocated to the cytoplasm from the nucleus in a Jab1-dependent manner (14). Jab1 is a member of CSN and functions as a platform to recruit CSN-associated kinases (21). We therefore used curcumin, a $\mathrm{CSN}$-associated kinase inhibitor, to test whether CSNdependent phosphorylation is involved in Jab1-mediated p53 regulation $(21,22)$. When p53 is expressed in p53-null H1299 cells, around $90 \%$ of the cells have p53 localized in the nucleus, while cells with both nuclear and cytoplasmic p53 had less than $10 \%$ (Fig. 1A, panels 1-4). Jab1 was found to be expressed in both the cytoplasmic and nuclear areas (Fig. 1A, panels 5-8). When Jab1 was co-expressed with p53, about $70 \%$ of the cells exhibited p53 nuclear/cytoplasmic localization, as previously observed (Fig. 1A, panels 9-12) (15). Interestingly, curcumin treatment significantly inhibited Jab1-mediated p53 cytoplasmic localization with only $16 \%$ of the cells displaying p53 in the cytoplasm (Fig. 1A, panels 13-16). To further confirm the effect of curcumin on Jab1-mediated p53 nuclear

ISSN: 1976-670X (electronic edition)

Copyright (C) 2017 by the The Korean Society for Biochemistry and Molecular Biology

(c) This is an open-access article distributed under the terms of the Creative Commons Attribution Non-Commercial License (http://creativecommons.org/licenses/by-nc/4.0) which permits unrestricted non-commercial use, distribution, and reproduction in any medium, provided the original work is properly cited. 

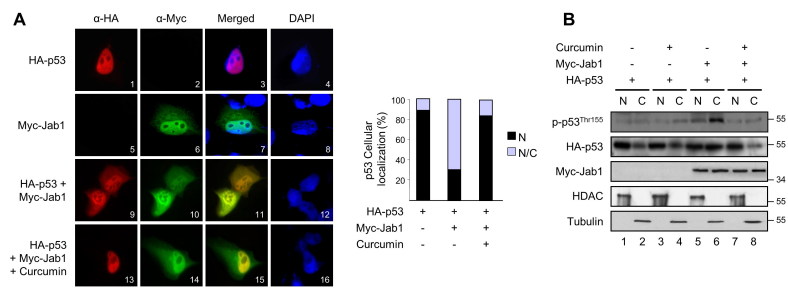

c
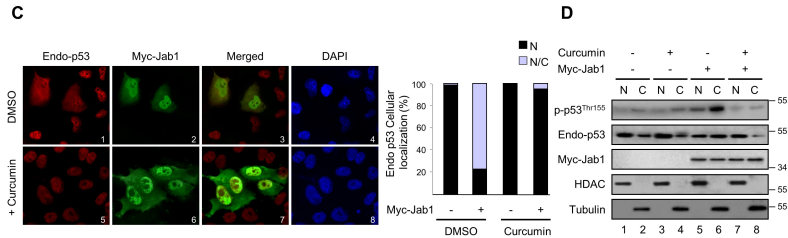

Fig. 1. Curcumin suppressed Jab1-mediated nuclear export of p53. (A) H1299 cells were transfected with the plasmids expressing HA-p53, Myc-Jab1, or both for $24 \mathrm{~h}$ and then treated with 50 $\mu \mathrm{M}$ curcumin for $6 \mathrm{~h}$. The cells were analyzed by fluorescence microscopy using anti-HA and Myc antibodies. The cells were counterstained with DAPI to visualize the nuclei. Representative images are shown in the left panel. A total of 200 cells expressing HA-p53 were counted according to their localization, and the results are presented in the right panel ( $\mathrm{N}$ : Nucleus, N/C: Nucleus and cytoplasm). (B) Nuclear and cytoplasmic fractions of H1299 cells transfected and treated as described in (A) were prepared using an NE-PER extraction kit. Protein levels were determined by western blot (WB) using anti-phospho-p53 (Thr155), $\mathrm{HA}$, or Myc antibodies. HDAC and tubulin were used as loading controls for the nuclear and cytoplasmic proteins, respectively. (C) U2Os cells transfected with the plasmid expressing HA-Jab1 were treated with DMSO or $50 \mu \mathrm{M}$ curcumin for $6 \mathrm{~h}$. The cells analyzed by fluorescence microscopy as in (A). (D) Nuclear and cytoplasmic fractions of U2OS cells transfected and treated as described in (C) were prepared using NE-PER extraction kit. Protein levels were determined by WB.

export, the nucleus and cytoplasm of cells were fractionated and analyzed by western blotting. Cytoplasmic p53 expression was found to be increased by Jab1 (Fig. 1B, lanes 2 and 6), whereas curcumin was found to have reduced p53 expression in the cytoplasm (Fig. 1B, lanes 6 and 8); both results are in support of the immunofluorescence data.

CSN complexes induce p53 phosphorylation at S149, T150, and T155 via CSN-associated kinases (21). Since Jab1 is a component of CSN complexes, we wondered whether Jab1 itself is able to stimulate phosphorylation of p53 in our system. The co-expression of Jab1 with p53 increased phosphorylation at T155, particularly in the cytoplasm (Fig. 1B, lane 6), and this process was abrogated by curcumin (Fig. 1B, lanes 6 and 8).

We next assessed the effects of curcumin on the Jab1mediated p53 nuclear export in the U2OS osteoblastoma cell line harboring intact p53. Notably, only Jab1-expressing cells exhibited p53 cytoplasmic localization, while cells without Jab1 showed p53 nuclear localization (Fig. 1C, panels 1-4). However, most of the curcumin-treated cells had p53 in the nucleus, regardless of Jab1 expression (Fig. 1C, panels 5-8).

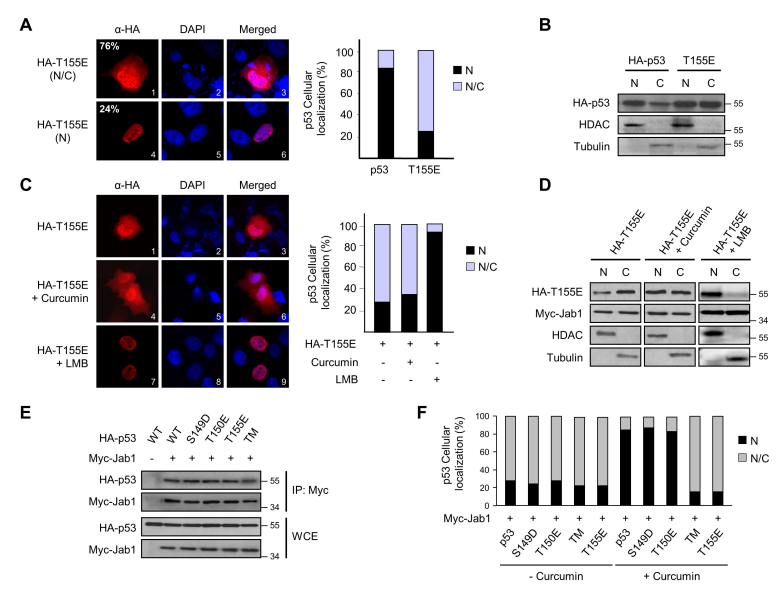

Fig. 2. Phosphomimetic mutant p53 (T155E) is mainly localized in the cytoplasm, in a CRM-dependent manner. (A) H1299 cells transfected with the plasmids expressing HA-p53 WT or T155E were analyzed by fluorescence microscopy. Representative images are shown and summarized in the right panel. (B) Nuclear and cytoplasmic fractions of H1299 cells expressing HA-p53 WT and T155 were subjected to WB. (C, D) H1299 cells expressing HA-p53 T155 were treated with $50 \mu \mathrm{M}$ curcumin for $6 \mathrm{~h}$ or 10 $\mathrm{nM}$ leptomycin B (LMB) for $12 \mathrm{~h}$. Immunofluorescence $(\mathrm{C})$ and WB (D) analysis were performed as described above. (E) H1299 cells were transfected with the plasmid expressing Jab1 and p53 wild-type (WT) or mutants. Whole cell extracts (WCE) were immunoprecipitated with an anti-Myc antibody, followed by WB using anti-HA and Myc antibody. (F) H1299 cells were transfected as indicated, then treated with or without $50 \mu \mathrm{M}$ curcumin for 6 h. The cells analyzed by fluorescence microscopy. Representative images are shown in the supplementary Fig. 2.

Cell fractionation analysis further supported our finding that ectopic Jab1 expression induced p53 accumulation in the cytoplasm, and this was reversed by curcumin treatment (Fig. 1D). Consistent with Fig. 1B, phospho-p53 was mostly found in the cytoplasmic fraction, but cytoplasmic phospho-p53 was mostly disappeared in the presence of curcumin (Fig. 1D). Overall, our data suggests that Jab1 stimulates p53 phosphorylation at Thr155 by $\mathrm{CSN}$-associated kinase, leading to p53 nuclear export.

\section{Phosphorylation of Thr 155 on p53 is required for Jab1-mediated p53 nuclear export}

As Jab1 induced p53 phosphorylation at Thr155 and cytoplasmic localization (Fig. 1 and Supplementary Fig. 1A), we next wondered whether p53 phosphorylation is sufficient for p53 nuclear export. To test this, we constructed S149D, T150E, T155E, and S149D/T150E/ T155E (triple-point mutant; TM) as p53 phospho-mimetics. When each mutant was expressed in cells, there was no difference among wild-type (WT) p53, S149D and T150E in their cellular distribution (Supplementary Fig. 1B). Interestingly, around $75 \%$ of cells expressing T155E or TM have p53 in the cytoplasm (Fig. 2A and Supplementary Fig. 1B). Cellular fractionation analyses 
showed that while WT p53 was mostly detected in the nucleus, $50 \%$ of $\mathrm{T} 155 \mathrm{E}$ was found in the cytoplasmic fractions (Fig. 2B). In particular, the spontaneous cytoplasmic localization of T155E was not inhibited by curcumin (Fig. 2C and D), but was suppressed by leptinomycin B (LMB), an inhibitor of the nuclear export system (Fig. 2C and D). These results suggest that cytoplasmic localization of T155E is no longer controlled by CSN-kinases, but requires a CRM1-dependent nuclear export system.

We next investigated the effect of Jab1 on the p53 mutants. All these mutants were able to bind to Jab1 (Fig. 2E). When Jab1 was co-expressed with S149D and T150E, their nuclear export pattern was similar to wild-type p53, and was inhibited by curcumin, suggesting that S149 and T150 on p53 are dispensable for Jab1-mediated p53 translocation (Fig. 2F and Supplementary Fig. 2, panels 1-12 and 21-32). On the other hand, T155E and TM, co-expressed with Jab1, displayed around $75 \%$ of cytoplasmic localization, which is similar to the spontaneous cytoplasmic localization, indicating that they were little affected by Jab1 (Fig. 2F and Supplementary Fig. 2, panels 13-20 and 33-40). These data suggest that the nuclear export of p53 is influenced by the change of electronic charge on Thr155 that occurs upon phosphorylation, and not by Jab1. In summary, Jab1 was able to stimulate phosphorylation of p53 at Thr155. The mutant, T155E, which mimics the charged structure of phosphorylated p53, was able to localize to the cytoplasmic area spontaneously in a CRM1-dependent manner.
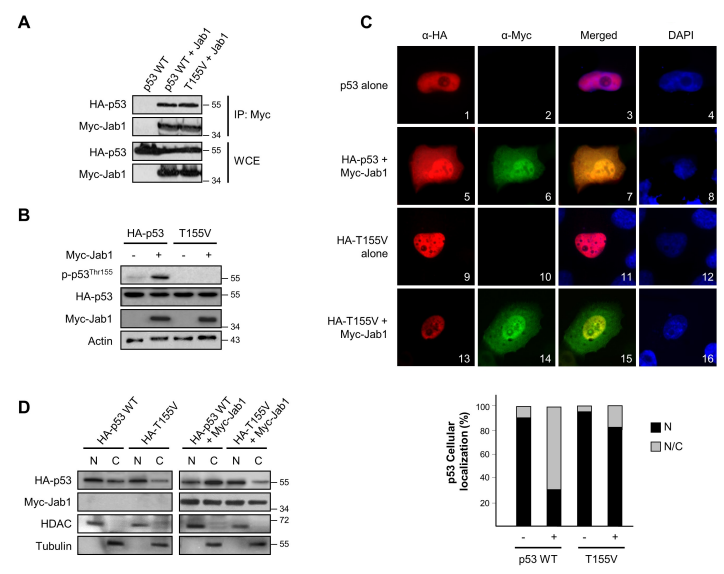

Fig. 3. The Thr155 residue on p53 are indispensable for Jab1mediated cytoplasmic localization and degradation. (A) Extracts from H1299 cells transfected as indicated were immunoprecipitated with an anti-Myc antibody, followed by WB using anti-HA and Myc antibody. (B) H1299 cells transfected with the plasmids expressing HA-p53 WT and T155V in the absence and presence of Myc-Jab1. Protein levels were determined by western blot (WB). (C) H1299 cells transfected with the indicated plasmids were analyzed by fluorescence microscopy. Representative images are shown and summarized in the right panel. (D) Nuclear and cytoplasmic fractions of $\mathrm{H} 1299$ cells transfected as indicated were subjected to WB.

\section{p53 T155V point mutant is resistant to Jab1-mediated nuclear export}

Since T155E is effectively localized into the cytoplasm even without overexpressed Jab1, the T155V mutant was constructed to determine whether Thr155 is indeed crucial for Jab1-mediated transport of p53. Despite its ability to bind to Jab1, Jab1-induced phosphorylation of T155V mutant was not observed (Fig. 3A and B). Using immunofluorescence analyses, we found that Jab1-mediated nuclear export of T155V was almost completely prevented (Fig. 3C, panels 5-8 and 13-16, graph). Cellular fractionation assays showed that only small fraction of T155V was observed in the cytoplasm, in the presence of Jab1 (Fig. 3D). Overall, the data showed that export of the p53 mutant defective in phosphorylation at Thr155 residue to the cytoplasm was prevented by Jab1, suggesting that the phosphorylation at Thr155 residue might be essential for p53 nuclear export.

\section{Phosphorylation at T155 of p53 is required for efficient nuclear export by $\mathrm{Hdm} 2$}

The canonical pathway of p53 cytoplasmic translocation requires $\mathrm{Hdm} 2$-mediated mono-ubiquitination at the C-terminal six lysine sites, followed by CRM1-dependent nuclear export $(23,24)$. The mutation or deletion of these sites prevents Hdm2-mediated nuclear export of p53. To examine whether the Hdm2-mediated pathway is involved for the cytoplasmic localization or T155E, we constructed a T155E6KR mutant in which Thr155 mutated to Glu, and six lysines to arginines.

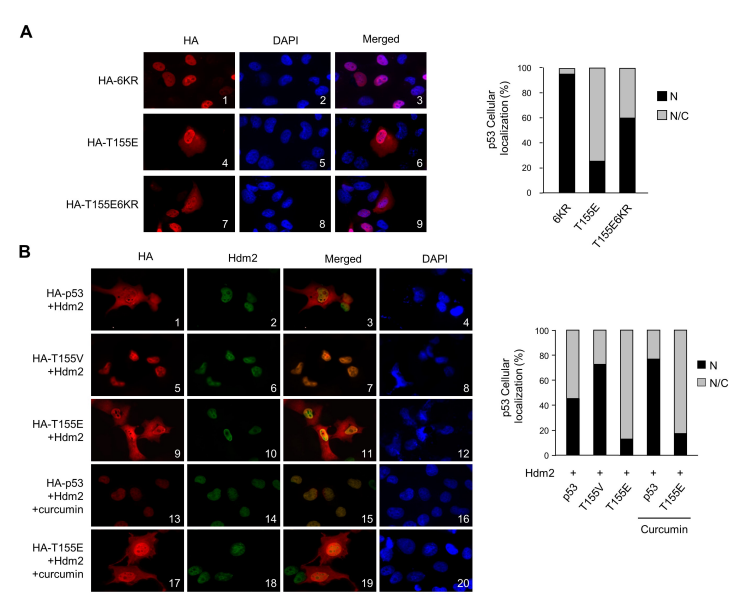

Fig. 4. Phosphorylation at the Thr155 residue and ubiquitination at the six lysine residues on p53 cooperatively facilitate cytoplasmic localization. (A) H1299 cells transfected with HA-p53 6KR, T155E, or T155E6KR were analyzed by fluorescence microscopy. Representative images are shown and summarized in the right panel. (B) H1299 cells were transfected with the plasmids expressing $\mathrm{HA}-\mathrm{p} 53$ and $\mathrm{Hdm} 2$, and then treated with $50 \mu \mathrm{M}$ curcumin for $6 \mathrm{~h}$. The cells were analyzed by fluorescence microscopy. Representative images are shown, and summarized in the right panel. 
While most cells expressing the 6KR mutant displayed p53 at the nucleus, $37 \%$ of cells expressing T155E6KR exhibited cytoplasmic p53 localization, suggesting that the p53 phospho-mimetic mutant could be exported to the cytoplasm in the absence of the C-terminal six lysines (Fig. 4A, panels 1-6). However, the nuclear export of T155E6KR was less efficient compared to that of T155E, implying that Hdm2 pathway might facilitate nuclear export of T155E (Fig. 4A, panels 4-9).

Accordingly, we employed p53/Mdm2 double knockout (DKO) mouse embryonic fibroblast (MEF) to exclude the effects of endogenous Mdm2. Cytoplasmic T155E was detected in $30 \%$ of cells expressing T155E when expressed in p53/Mdm2 DKO MEFs, suggesting that T155E could be exported to cytoplasm independent of Mdm2 (Supplementary Fig. 3). In addition, Hdm2 further enhanced cytoplasmic localization of T155E mutant even better than that of WT p53 (Supplementary Fig. 3).

Next, the effects of Hdm2 on p53 mutants were tested. The data showed that $\mathrm{Hdm} 2$ was able to induce nuclear export of WT p53 in $55 \%$ of cells (Fig. 4B, panels $1-4$, graph). The Hdm2-mediated translocation efficiency dropped to $25 \%$ for the T155V mutant, while it increased to $90 \%$ for T155E (Fig. $4 \mathrm{~B}$, panels $5-12$, graph). This indicates that the simultaneous mono-ubiquitination at the C-terminus and phosphorylation at Thr155 can independently stimulate nuclear export of p53. Further, curcumin was able to suppress Hdm2-mediated nuclear export of p53, whereas it did not inhibit that of the T155E mutant (Fig. 4B, panels 13-20, graph). This data suggests that the inhibitory effect of curcumin on Hdm2-mediated p53 nuclear export might be due to suppression of p53 phosphorylation at Thr155. In conclusion, Jab1-mediated p53 phosphorylation at Thr155 can induce the nuclear export of p53 independently of $\mathrm{Hdm} 2$, but this process can be further facilitated by Hdm2 overexpression.

\section{DISCUSSION}

Cytoplasmic localization of p53 is implicated in two pathways; one of which carries p53 into the cytoplasm and mitochondria to induce apoptosis; the other disables the nuclear transcriptional activity of p53, leading to cell survival. The major pathway that stimulates p53 cytoplasmic localization is mediated by the $\mathrm{Hdm} 2$-dependent ubiquitination of p53 at the six lysines (K370, K372, K373, K381, K382, and K386) located at the C-terminus $(24,25)$. Hdm2 plays a dual role in a dose-dependent manner when negatively regulating p53. Firstly, if the levels of Hdm2 are low, perhaps due to low levels of p53, monoubiqutination of p53 results $(24,26)$. This leads to exposure of leucine-rich nuclear export signal (NES) located on the C-terminal end and subsequent detachment of Hdm2 from the p53 $(23,24)$. Monoubiquitination would allow the interaction between p53 and the nuclear export system containing CRM1 and RanGTP, which will induce nuclear export of p53 $(24,27)$. Supporting this mechanism, p53 artificially labeled with ubiquitin is exported to the cytoplasm $(24,26)$. Hdm2 mutant defective in cytoplasmic localization is still able to induce p53 nuclear export $(28,29)$. This suggests that $\mathrm{Hdm} 2$ might not be physically responsible for the delivery of p53 into the cytoplasm. Once localized to the cytoplasm, p53 can be polyubiquitinated by other E3 or E4 ligases, and eventually degraded by the cytoplasmic $26 \mathrm{~S}$ proteasome (26, 27). Otherwise, it could be also involved in mitochondrial apoptotic pathways $(30,31)$. Secondly, when the levels of $\mathrm{Hdm} 2$ are high, polyubiquitination of p53 and subsequent nuclear degradation is induced by $\operatorname{Hdm} 2(24,26,27)$. The post-translational modifications that affect cellular localization of p53 also include phosphorylation at Thr55 of p53 (31). This process increased the binding of p53 with CRM1, facilitating its translocation into the cytoplasm. Recent studies suggest that $\mathrm{Hdm} 2-$ mediated ubiquitination reduces $\mathrm{Hdm} 2$ and p53 affinity, which enable the mono-ubiquitinated p53 to be localized to the cytoplasm. In this pathway, sumoylation by PIASy boosted this process (24). Another report suggested that phosphorylation at Thr55 was able to induce Hdm2-independent cytoplasmic localization by increasing its affinity toward CRM1 (31). This suggests the existence of a possible non-conventional mechanism leading p53 to the cytoplasm. On the other hand, nuclear export of p53 can be blocked upon DNA damaging stresses. Post-translational modifications of p53 such as phosphorylation, acetylation, glycosylation, hydroxylation, methylation, adenylation, etc. could lead to the suppression of Hdm2-mediated ubiquitination of p53 $(27,30,32)$.

In a previous study, we showed that Jab1, a component of CSN complexes, was able to induce nuclear export of p53 (15). In this report, we further showed that Jab1 was able to stimulate phosphorylation of p53 at Thr155 via CSNassociated kinases as curcumin, an inhibitor of CSN-associated kinases, blocked this process. Once phosphorylated, p53 seems to translocate into the cytoplasm by itself, since T155E could be localized in the cytoplasm in Mdm2-deficient cells or in the presence of curcumin. However, LMB, an inhibitor of CRM1-dependent nuclear export, abrogated the cytoplasmic T155E localization, suggesting that phosphorylation of p53 at Thr155 residue might facilitate the interaction with the CRM complex. In addition, cytoplasmic localization of T155E is further enhanced by $\mathrm{Hdm} 2$ overexpression, implying that Hdm2-mediated mono-ubiquitination of p53 and Jab1mediated p53 phosphorylation cooperatively increases the interaction between CRM1 and p53, which ultimately facilitates nuclear export of p53.

Despite increased cytoplasmic localization of the T155E or TM mutant, their transcriptional activity was similar to WT p53, suggesting that the remaining nuclear p53 is sufficient to induce its transcriptional activity (Supplementary Fig. 4A). Further, Jab1 could suppress the transcriptional activity of p53 mutants (Supplementary Fig. 4A). Interestingly, the activity of T155V mutant, whose localization was not changed by Jab1, 
was inhibited by Jab1 (Supplementary Fig. 4B). These results imply that Jab1 can suppress the transcriptional activity of p53, independently of subcellular localization of p53, and possibly via direct binding.

In summary, our studies identified a detailed mechanism of p53 nuclear export mediated by CSN/Jab1-mediated phosphorylation and Hdm2-mediated ubiquitination. These observations may provide an effective approach to p53-activating anti-cancer therapy.

\section{MATERIALS AND METHODS}

\section{Plasmids}

pcDNA3-HA-p53 (wild-type, 6KR), pCS-MT-BX-Myc-Jab1, pCMVHdm2, and PG13-luc were used as previously described (15, 33). p53 point mutants, S149D, T150E, T155E, TM, and T155V, were generated by site-directed mutagenesis.

\section{Cell culture and DNA transfection}

p53/Mdm2 DKO MEFs were kindly provided by G. Lozano (Univ. of Texas). H1299 (p53 null Lung carcinoma), U2OS (p53-positive Osteosarcoma), and p53/Mdm2 DKO MEFs were maintained in Dulbecco's modified Eagle's Medium (DMEM, Hyclone) supplemented with $10 \%$ fetal bovine serum (Hyclone) and $1 \%$ penicillin/streptomycin (Invitrogen). Transient transfections were performed using Lipofectamine 2000 (Invitrogen), according to the manufacturer's recommendations.

\section{Antibodies and chemicals}

The following antibodies were used: Jab1 (sc-9074, FL-334), Mdm2 (sc-965, SMP-14), p53 (sc-6243, FL-393), p53 (sc-126, DO-1), Phospho-p53 (Thr155, sc-17105), HA mouse (sc-7932, F-7), HA rabbit (sc-805, Y-11), and Myc (sc-40, 9E10) antibodies from Santa Cruz Biotechnology (Santa Cruz, CA). $\beta$-Actin antibodies from Sigma (A5316). PARP from Cell Signaling (9542). Alexa Fluor 488 anti-mouse and Alexa 594 anti-rabbit antibody were purchased from Alexa. Leptomycin B was purchased from Sigma.

\section{Immunofluorescence staining}

Immunofluorescence assays were performed as described previously $(14,34)$. Briefly, cells were transfected using lipofectamine 2000 for $24 \mathrm{~h}$, fixed with $4 \%$ paraformaldehyde, and permeabilized with $0.5 \%$ Triton X-100. The cells were then blocked with $5 \%$ bovine serum albumin (BSA) in PBS, incubated with the specific primary antibody, and then incubated with Alexa Fluor 488 anti-mouse or Alexa 594 anti-rabbit antibodies. The cells were counterstained with DAPI (4, 6-diamidino-2-phenylindole). The slides were analyzed using confocal or immunofluorescent microscopes.

\section{Immunoprecipitation and Western blot analysis}

Immunofluorescence assays were performed as described previously $(34,35)$. Briefly, cells were lysed in IP buffer (50
$\mathrm{mM}$ Tris- $\mathrm{HCl} \mathrm{pH} 7.5,150 \mathrm{mM} \mathrm{NaCl}, 0.5 \%$ NP40, 0.5\% Triton $\mathrm{X}-100,0.1 \%$ sodium deoxycholate, and $1 \mathrm{mM}$ EDTA containing a protease inhibitor cocktail). Whole cell extracts were immunoprecipitated by incubation with antibodies for 2 $\mathrm{h}$ or overnight, followed by incubation with protein $\mathrm{A} / \mathrm{G}$ beads for $2 \mathrm{~h}$. Immunoprecipitates were boiled in 2x sample buffer for $5 \mathrm{~min}$. The extracts were separated by SDS-PAGE and analysed by western blot using the antibodies.

\section{Luciferase assay}

Luciferase activities were measured using the Dual-Luciferase assay kit (Promega) according to the manufacturer's instructions. The Renilla luciferase reporter plasmid, pRL-CMV, was used as an internal control.

\section{ACKNOWLEDGEMENTS}

This research was supported by grants from the National Research Foundation of Korea (NRF) funded by the Ministry of Education (NRF-2012R1A6A3A04040105) and by the Ministry of Science, ICT and Future Planning (NRF-2016R1C1B2013430 and NRF-2015M3A9B5030308).

\section{CONFLICTS OF INTEREST}

The authors have no conflicting financial interests.

\section{REFERENCES}

1. Campisi J (2005) Senescent cells, tumor suppression, and organismal aging: good citizens, bad neighbors. Cell 120, 513-522

2. Lane DP (1992) Cancer. p53, guardian of the genome. Nature 358, 15-16

3. Levine AJ (1997) p53, the cellular gatekeeper for growth and division. Cell 88, 323-331

4. Vousden KH and Lu X (2002) Live or let die: the cell's response to $p 53$. Nat Rev Cancer 2, 594-604

5. Haupt Y, Maya R, Kazaz A and Oren M (1997) Mdm2 promotes the rapid degradation of p53. Nature 387, 296-299

6. Kubbutat $\mathrm{MH}$, Jones $\mathrm{SN}$ and Vousden $\mathrm{KH}$ (1997) Regulation of p53 stability by Mdm2. Nature 387, 299-303

7. Charni M, Aloni-Grinstein R, Molchadsky A and Rotter V (2017) p53 on the crossroad between regeneration and cancer. Cell Death Differ 24, 8-14

8. Roth J, Dobbelstein M, Freedman DA, Shenk T and Levine AJ (1998) Nucleo-cytoplasmic shuttling of the hdm2 oncoprotein regulates the levels of the p53 protein via a pathway used by the human immunodeficiency virus rev protein. EMBO J 17, 554-564

9. Wei $N$ and Deng XW (1999) Making sense of the COP9 signalosome. A regulatory protein complex conserved from Arabidopsis to human. Trends Genet 15, 98-103

10. Tomoda K, Kubota Y, Arata Y et al (2002) The cytoplasmic 
shuttling and subsequent degradation of p27Kip1 mediated by Jab1/CSN5 and the COP9 signalosome complex. J Biol Chem 277, 2302-2310

11. Doronkin S, Djagaeva I and Beckendorf SK (2003) The COP9 signalosome promotes degradation of Cyclin $\mathrm{E}$ during early Drosophila oogenesis. Dev Cell 4, 699-710

12. Kim BC, Lee HJ, Park SH et al (2004) Jab1/CSN5, a component of the COP9 signalosome, regulates transforming growth factor beta signaling by binding to Smad7 and promoting its degradation. Mol Cell Biol 24, 22512262

13. Callige M, Kieffer I and Richard-Foy H (2005) CSN5/Jab1 is involved in ligand-dependent degradation of estrogen receptor $\{$ alpha\} by the proteasome. Mol Cell Biol 25, 4349-4358

14. Lee EW, Oh W and Song J (2006) Jab1 as a mediator of nuclear export and cytoplasmic degradation of p53. Mol Cells 22, 133-140

15. Oh W, Lee EW, Sung YH et al (2006) Jab1 induces the cytoplasmic localization and degradation of p53 in coordination with Hdm2. J Biol Chem 281, 17457-17465

16. Oh W, Yang MR, Lee EW et al (2006) Jab1 mediates cytoplasmic localization and degradation of West Nile virus capsid protein. J Biol Chem 281, 30166-30174

17. Kim JH, Choi JK, Cinghu S et al (2009) Jab1/CSN5 induces the cytoplasmic localization and degradation of RUNX3. J Cell Biochem 107, 557-565

18. Guo H, Jing L, Cheng Y et al (2016) Down-regulation of the cyclin-dependent kinase inhibitor p57 is mediated by Jab1/Csn5 in hepatocarcinogenesis. Hepatology 63, 898-913

19. Lu R, Hu X, Zhou J et al (2016) COPS5 amplification and overexpression confers tamoxifen-resistance in ERalphapositive breast cancer by degradation of NCoR. Nat Commun 7, 12044

20. Tomoda K, Yoneda-Kato N, Fukumoto A, Yamanaka S and Kato JY (2004) Multiple functions of Jab1 are required for early embryonic development and growth potential in mice. J Biol Chem 279, 43013-43018

21. Bech-Otschir D, Kraft R, Huang $X$ et al (2001) COP9 signalosome-specific phosphorylation targets p53 to degradation by the ubiquitin system. EMBO J 20, 16301639

22. Yang WH, Kim JE, Nam HW et al (2006) Modification of p53 with O-linked $\mathrm{N}$-acetylglucosamine regulates p53 activity and stability. Nat Cell Biol 8, 1074-1083

23. Stommel JM, Marchenko ND, Jimenez GS, Moll UM, Hope TJ and Wahl GM (1999) A leucine-rich nuclear export signal in the p53 tetramerization domain: regulation of subcellular localization and p53 activity by NES masking. EMBO J 18, 1660-1672

24. Carter S, Bischof O, Dejean A and Vousden KH (2007) C-terminal modifications regulate MDM2 dissociation and nuclear export of p53. Nat Cell Biol 9, 428-435

25. Lohrum MA, Woods DB, Ludwig RL, Balint E and Vousden KH (2001) C-terminal ubiquitination of p53 contributes to nuclear export. Mol Cell Biol 21, 85218532

26. Li M, Brooks CL, Wu-Baer F, Chen D, Baer R and Gu W (2003) Mono-versus polyubiquitination: differential control of p53 fate by Mdm2. Science 302, 1972-1975

27. Coutts AS, Adams CJ and La Thangue NB (2009) p53 ubiquitination by Mdm2: a never ending tail? DNA Repair (Amst) 8, 483-490

28. Boyd SD, Tsai KY and Jacks T (2000) An intact HDM2 RING-finger domain is required for nuclear exclusion of p53. Nat Cell Biol 2, 563-568

29. Geyer RK, Yu ZK and Maki CG (2000) The MDM2 RING-finger domain is required to promote p53 nuclear export. Nat Cell Biol 2, 569-573

30. Dai C and Gu W (2010) p53 post-translational modification: deregulated in tumorigenesis. Trends Mol Med 16, 528-536

31. Cai X and Liu X (2008) Inhibition of Thr-55 phosphorylation restores p53 nuclear localization and sensitizes cancer cells to DNA damage. Proc Natl Acad Sci U S A 105, 16958-16963

32. Brooks CL and Gu W (2006) p53 ubiquitination: Mdm2 and beyond. Mol Cell 21, 307-315

33. Lee EW, Lee MS, Camus S et al (2009) Differential regulation of p53 and p21 by MKRN1 E3 ligase controls cell cycle arrest and apoptosis. EMBO J 28, 2100-2113

34. Seo J, Lee EW, Sung H et al (2016) CHIP controls necroptosis through ubiquitylation- and lysosomedependent degradation of RIPK3. Nat Cell Biol 18, 291-302

35. Lee EW, Kim JH, Ahn YH et al (2012) Ubiquitination and degradation of the FADD adaptor protein regulate death receptor-mediated apoptosis and necroptosis. Nat Commun 3, 978 